\title{
VERHALTENHEIT: LA TONALIDAD DE UN POSIBLE NUEVO INICIO HISTÓRICO
}

\author{
Valentina Bulo Vargas \\ Universidad Austral de Chile \\ valenbulo@hotmail.com
}

\begin{abstract}
Resumen
En el pensar heideggeriano, tanto en su época temprana como tardía, ha habitado la idea una "máscara" respecto a una "figura originaria", ya sea como propiedad e impropiedad, o como el abandono del ser en el inicio del pensar occidental respecto a un despliegue originario del ser como Ereignis; la principal labor filosófica de Heidegger se ha abocado a remover las capas y así deconstruir el camino trazado por la metafísica; un pensamiento vivo que "piensa para atrás". Sin embargo este no es el único modo en que Heidegger "ejerce" pensamiento, pues junto al "paso atrás", Heidegger nos indica a veces y de un modo siempre sugerente, la posibilidad del salto. Ambos movimientos del pensar están configurados desde experiencias afectivas concretas, las que no son subjetivas sino el trazo mismo de cada figura epocal del pensamiento-ser, del Ereignis. Atenderé en este escrito únicamente al temple fundamental sugerido por Heidegger para el segundo inicio en los Beiträge zur Philosophie: la reserva (Verhaltenheit), para luego indicar desde ella hacia lo que en sus escritos últimos llamará desasimiento (Gelassenheit).
\end{abstract}

Palabras Clave: Heidegger, ser, pensar, desasimiento.

\section{Abstract}

In Heidegger's thinking, both in its earlier as in its later stages, the idea lives under a "mask" which stands to an "original figure" either as the improper in relation to what is proper, or as the defection from being at the beginning of Western thought in relation to the original desplay of being as Ereignis. Heidegger's main philosophical job was to remove the masking layers and thereby to deconstruct the route travelled by metaphysics: his is a living thought that "thinks backwards". However, this is not the only way in which Heidegger "practices" thinking: together with "stepping back", Heidegger often suggests the possibility of "jumping". Both motions of thought start from concretely experienced moods, which are not subjective but constitute the very trail of each epochal figure of thought-being, of Ereignis. In this essay I only pay attention to the fundamental mood proposed by Heidegger for the second beginning in Beiträge zur Philosophie: namely, reticence (Verhaltenheit); but I point thence to what in his last writings he called detachment (Gelassenheit).

KeYwords: Heidegger, being, thinking, detachment. 
$\overline{R A}$ En el pensar heideggeriano, tanto en su época temprana como tardía, ha habitado la idea una "máscara" respecto a una "figura originaria", ya sea como propiedad e impropiedad, o como el abandono del ser en el inicio del pensar occidental respecto a un despliegue originario del ser como Ereignis; la principal labor filosófica de Heidegger se ha abocado a remover las capas y así deconstruir el camino trazado por la metafísica; un pensamiento vivo que "piensa para atrás". Sin embargo, este no es el único modo en que Heidegger "ejerce" pensamiento, pues junto al "paso atrás", Heidegger nos indica a veces y de un modo siempre sugerente, la posibilidad del salto. Ambos movimientos del pensar están configurados desde experiencias afectivas concretas, las que no son subjetivas, sino el trazo mismo de cada figura epocal del pensamiento-ser, del Ereignis. Atenderé en este escrito únicamente al temple fundamental sugerido por Heidegger para el segundo inicio en los Beiträge zur Philosophie: la reserva (Verhaltenheit), para luego indicar desde ella hacia lo que en sus escritos últimos llamará desasimiento (Gelassenheit).

El segundo inicio es el despliegue del Ser propiamente como Ereignis. Esto nos indica, en primer lugar, que Ereignis es un modo originario del despliegue del Ser. Eso es lo distinto respecto a los otros modos epocales en donde el Ser se retira. La originariedad del Ereignis de algún modo absorbe a los otros modos epocales del ser y "late" tras cada uno de ellos, por eso no es un modo completamente otro del retiro del Ser y en este sentido se identifican. De hecho, toda la interpretación ontohistórica de Heidegger, me parece, pretende absorber (situarse "tras") las interpretaciones tradicionales y el ángulo desde donde se interpretan es desde el Ser como Ereignis. Hay modos epocales porque el Ser se despliega, es puro acontecer; hay retiro, porque hay Ereignis. Por esta razón hemos de evitar aquí una lectura de otro inicio "completamente otro", vale decir, concebirlo meramente a diferencia del primero.

Todo inicio ha de surgir de una "necesidad del ser", entendida ésta, a diferencia de una necesidad lógica, al modo de un apremio. Este apremio se efectúa a partir de un temple fundamental, el que no está determinado por el apremio como tal temple en particular sino solo "forzado" por él. El temple fundamental es un modo efectivo del apremio entre otros temples fundamentales posibles. Aquí se funda la posibilidad de otro inicio, otro modo posible de desplegarse el Ser. Del apremio no se sigue necesariamente el asombro, que es el temple rector del primer inicio del pensar occidental. Es en esta indeterminación del apremio donde Heidegger fundamentará un despliegue originario del Ser como Ereignis en un posible "otro inicio". Ahora la cuestión es determinar qué temple es capaz de emprender un inicio y que además configure el modo del despliegue del ser como Ereignis, fundando con ello al Dasein y restituyendo al Ser en el ente.

Si bien "la época en donde ha de edificarse la figura esencial del ente a partir de la verdad del Ser no ha llegado todavía" (Heidegger 1989, p. 5), Heidegger intentará "indicar" hacia él, sugerirlo, desde su resonar en el abandono, en este sentido, "todo meditar sobre este temple es siempre solo un preparativo paulatino para la incidencia atemperante (stimmender Einfall) del temple fundamental" (Heidegger 1989, p. 22). Este temple será nombrado como la reserva. 
La reserva es el temple que permitirá el despliegue originario del Ser como Ereignis y como tal ha de reconfigurar el modo de ensamblarse la articulación del Ser respecto al ente, al hombre y los dioses; es decir, de fundar un nuevo espacio de tiempo. Para intentar comprender esto y, sobre todo, para tener los elementos que nos permitan "ver" si efectivamente la reserva "puede" cumplir esta función, nos acercaremos primeramente a una acotación de lo que es la reserva misma y luego nos dirigiremos a este nuevo espacio de tiempo, parcelando su iniciarse en dos momentos: la articulación de "piezas" de este nuevo ensamble (el Entre Dasein-Ser y ente), y el insertarse de una de estas piezas, el Dasein, en la articulación.

A) LA RESERVA CONSIDERADA EN Sí MISMA: El término alemán (Verhaltenheit) es traducido también como retención, contención. Indica primeramente cierto modo de tener (halten), un re-tener. El problema está en cómo entendemos este tener. Si entendemos la contención transitivamente hay que preguntarse por el contenido, se contiene "algo", como el vaso al agua. La reserva sería el momento de contención del Ser; o de retención, como cuando retenemos a alguien. Pero ¿el Ser se entiende como continente o contenido? Podemos decir por ahora que este problema se sitúa más allá de estas categorías; no se trata de que el Dasein contenga al Ser o viceversa, ni tampoco de que "se contengan" entre sí.

A lo mejor, el término sugiere una cierta reflexividad en el sentido de contenerse, com-portar-se, re-tener-se; rápidamente podemos ser llevados a interpretar la reserva desde la clásica "continencia", y afirmar que la reserva es una continencia del Dasein, por ejemplo, respecto al mundo técnico; contención aquí mienta una demarcación de límites. Tradicionalmente, la continencia es un límite respecto a los apetitos e impulsos. Me parece que estas lecturas no son, si se ha de partir del Ereignis, líneas desde donde pueda surgir la comprensión de un temple fundamental que funde un inicio histórico. Es probable que en algún momento pueda haber continencia del Dasein en la reserva, pero no es lo primero.

Sin ser inexactas las traducciones de Verhaltenheit por retención o contención, he optado por el término reserva, pues se muestra de un modo más claro el sentido de la interpretación que haremos aquí de ella. El término reserva nos hace pensar en una reserva de agua, de vino, una reserva forestal, allí puede sonar mejor el hablar de una reserva del Ser, como un lugar de preservación del acontecer. En una reserva forestal, por ejemplo, hay contención, pero ésta se entiende como acogida y cuidado. La contención surge de una consistencia, y la reserva, me parece, se dirige a la preservación de esta consistencia, los límites no surgen de un modo violento, no son una represión; algo puede contener (una persona que contiene a otra en la tristeza, por ejemplo), porque tiene la consistencia para acoger, $y$, si se quiere hablar de contención, ha de entenderse en este sentido.

"Reserva: como el origen de la calma y como la norma de recogimiento. El recogimiento en la calma y el cobijamiento de la verdad. El cobijamiento de la verdad y su desplegarse en la preocupación y el trato" (Heidegger 1989, p. 35). La reserva es aquella calma, aquella quietud que permite recolectar, recopilar, recoger, reunir aquello 
que está disperso (disgregado) y con ello instaurar una norma de reunión, dejando así que se cobije la verdad del Ser en el ente. La reserva aparece aquí como un verdadero principio reunidor, como la apertura de un calmo espacio tiempo desde donde la verdad se despliega cobijadamente. Recordemos, se trata del cobijamiento de la verdad del Ser en el ente, es decir, un mismo momento en donde se restituye al ente, se cobija la verdad y se reconfigura un nuevo modo de relación del hombre con el Ser, en copertenencia. Todo esto desde aquel temple que posee la quietud necesaria para permitir "que todo vuelva a su sitio": al origen. La reserva definirá el estilo del pensar del segundo inicio, y define también un nuevo modo del trato con los entes, arraigados ya en el Ser. La reserva es el momento en que se ha de superar el abandono, si es que nos ha de ser otorgada esta posibilidad.

B) La ReServa Como el inicio de una nueva aRticulación: Como todo temple fundamental, la reserva no es un momento subjetivo del Dasein, no es una actitud del Dasein respecto al Ser, es un momento del Entre, que surge de un apremio, de una temperante necesidad. En este sentido, el temple instaura ciertos modos de la verdad, como un bosquejo antepredicativo desde donde toman posición el ser, el ente y el hombre, uno respecto al otro. Es justo un espacio de juego de tiempo, esta posición es la figura epocal del ser, que acontece propiamente en la dis-posición afectiva. Desde distintos temples, la verdad se configura también distintamente y, por ejemplo, el mismo "rigor" de la reserva es constitutivamente distinto al de la "exactitud" que busca la certeza moderna. Seguramente el rigor de la reserva no violenta el ser en las cosas, como la exactitud, lo deja permanecer en ellas y permite que se "muestre" en lo oculto su acontecer.

En términos más técnicos: si el "Entre se despliega entre nosotros y el Ser, y si este mismo Entre pertenece al despliegue del Ser" (Heidegger 1989, p. 368), el temple situado en el Entre será una trama originaria, desde donde se configura el modo de la verdad. El instante en donde esto acontece, en el caso de la reserva será en la experiencia del Dasein como Dasein, es decir, como perteneciente al Ser. Es allí donde se "inserta" el Dasein como tal en la historia y "a una" se reconfigura la relación del ser como Ereignis, del hombre como Dasein y del ente como el ente restituido a partir del Ser. Este como alude al modo concreto del despliegue del Ser, "tramado", "ajustado" desde la figura concreta de un temple fundamental, en el caso del segundo inicio, de la reserva. Aquí se descubre la figura del Ereignis en la reserva como tal.

En efecto, "la reserva es la relación destacada e inmediata con el Ereignis" (Heidegger 1989, p. 31), como tal será la "apertura para la acallada cercanía del despliegue del Ser, que atemperando al lejanísimo temblor de señas que se acontecen desde la lejanía de lo indecidible" (Heidegger 1989, p. 31). Ahora bien, esto no quiere decir, por la concepción misma de Ereignis, que la reserva se abra a un ya configurado Ser, sino que funda, como soporte de un diseño, la pertenencia de Dasein y Ser como Ereignis, por eso es temple fundamental. "Ella es el temple-fundamental porque acuerda la profundización (Ergründung) del fundamento del Da-sein, del acontecimiento, y, con ello, la fundación del Dasein" (Heidegger 1989, p. 34). Esto significa que la reserva es quien atempera cabalmente y sostiene (durchstimmt und trägt) la fundación, 
y con ello, define el estilo del pensar en el segundo inicio, más bien dicho, es el estilo del pensar.

"De un simple impulso (Ruck) del pensar esencial tiene que ser traspuesta (versetzt) la verdad del Ser del primer inicio hacia el otro, para que en la sugerencia resuene la canción totalmente otra del Ser" (Heidegger 1989, pp. 8s). Este impulso trasponedor es propio de todo temple fundamental, y la reserva, en este sentido, tendrá el poder de "trasponer" el Entre, de dislocarlo, en el sentido de fundar un espacio de tiempo en donde se "relacionen" de un nuevo modo el hombre, el ser y los entes.

En el segundo inicio "la relación con el Ser, y de antes y de siempre, con la verdad del Ser, cambia en la forma de un dislocarse (Verrückung) hacia el Da-sein mismo... sobre todo en el otro inicio ha de llevarse a cabo el salto al interior del Entre" (Heidegger 1989, p. 14). Aquí, el Entre expresa la unidad originaria de Ser y Dasein en la verdad del Ser, la dislocación es el salto que abre de un nuevo modo esta unidad, propiamente como un Entre. Lo "dislocador" del salto es en rigor el Ser, pero si lo entendemos como Ereignis, como una copertenencia en donde el Ser se despliega y donde el "destello" de este despliegue coapropiador es el temple, lo dislocador es el temple fundamental, como lo afirma respecto al asombro, y ahora, en el otro inicio, respecto a la reserva. "Primeramente por el temple es medida la dimensión de la dislocación del Da-sein y le es atribuida la simplicidad del encanto (Berückung), en tanto se trate de la reserva como el temple de ánimo fundamental (Grund-stimmung)" (Heidegger 1989, p. 34).

C) La Reserva considerada desde el Dasein: Esta misma dislocación o desplazamiento, es analizada también desde el "respecto" del Dasein y como tal va a consistir en una inserción (Einrückung) en la apertura, que funda al Dasein en cuanto tal, pues se inserta en el desplazamiento. "El proyecto del $D a$-sein es posible sólo como inserción en el Da-sein. Pero el proyecto insertado surge únicamente a partir del ajuste (Fügsamkeit) de la juntura (Fügung) más oculta de nuestra historia en el temple fundamental de la reserva" (Heidegger 1989, p. 309). Aquí, la reserva aparece como aquel ajuste de la juntura (del Ser), el lugar donde se "ensambla", como inserción, el Dasein en su ser y con ello "en" la historia del Ser. Obviamente, esta inserción no es posterior al desplazamiento, "lo que" se desplaza, por decirlo de algún modo, es el ajuste originario.

La inserción es el modo concreto como acontece la trasposición desde el Dasein, y esta está dada como una "experiencia” del Dasein como Dasein. "El Dasein es experimentado, no re-presentado como objeto, sino cumplido y soportado como Da-sein, a través de una inserción desplazante (verrückende Einrückung)" (Heidegger 1989, p. 309). Experiencia no mienta aquí el aspecto subjetivo y particular del Dasein hacia el Ser, sino el momento en que se inaugura la apertura del lugar del Ser, eso es la fundación del Dasein, y en eso consiste la experiencia, en la apertura del espacio de un modo de despliegue. En el momento en que el Dasein se experimenta perteneciendo al Ser, es decir como Dasein, es cuando se abre este nuevo espacio tiempo capaz de emprender un inicio histórico. 
Desde esta perspectiva es que la reserva será "la" experiencia fundamental. Entendiendo experiencia desde la inserción, como un "ingresar". "El despliegue esencial es aquello en donde hemos de ingresar (einfahren). Eso es lo que quiere significar aquí "experiencia" (Erfahrung): ingresar en el despliegue, para que puestos en él, lo soportemos, lo que acontece en cuanto Da-sein y su fundación" (Heidegger 1989, p. 289). La experiencia del Dasein como Dasein es eo ipso su fundación, pues ella misma es inserción desplazante que configura, desde el Da-sein, el espacio de tiempo. Por eso, el temple fundamental es portador de un inicio, porque es el momento mismo en que se "corresponde" al Ser en el Dasein, por eso la reserva es experiencia fundamental. "La experiencia fundamental no es el enunciado... sino el detenerse de la reserva frente al recusarse reticente en la verdad (el claro del cobijamiento) del apremio... cuando esta reserva viene a palabra, lo dicho es siempre Ereignis" (Heidegger 1989, p. 80). Como experienciación del Dasein en cuanto tal, la reserva será la puesta en práctica de un modo de experiencia alejado de una re-presentación de la esencia general del hombre y pretenderá ser con ello el "momento" en que el Dasein se abra a su ser y se inserte de este modo en el despliegue de la verdad.

Y es que la reserva es experiencia del Dasein y con ello es también experiencia del Ser. En efecto, la reserva, a diferencia de todos los otros temples fundamentales que hemos visto, se templa ante el Ser mismo y no al ente en total, siente el Ser, por eso ella incluye al temple rector del presentir, llegando incluso a "definir" al hombre con esta caracterización: "El hombre presiente el Ser, es el presentidor del Ser (Ahnende des Seyns)" (Heidegger 1989, p. 245), con ello instaura una experiencia del Ser en el presentir, ya no se trata de sentir el ser "indirectamente" a través de la experiencia del ente (o de lo no-ente), sino que "junto a" los entes y "en" ellos se está templado al Ser en cuanto tal.

Es así como la reserva es la instancia (Inständigkeit) de inserción en el despliegue de la verdad, es el momento de "correspondencia" al Ser, pero sin entificarlo, sin suprimirlo, sino más bien dejando que sobresalga lo oculto del Ser. “¿Qué quiere decir 'estar parados' ('stehen') en el claro del ocultamiento y resistirlo? El temple fundamental de la reserva. Lo característico e históricamente único de esta insistencia (Inständigkeit), es que aquí se decide única y primeramente acerca de lo 'verdadero' "(Heidegger 1989, pp. 352s). El claro del ocultamiento es la esencia de la verdad, y precisamente la reserva es quien "está en" ella y la resiste. Como portadora de un inicio, la reserva "soporta", guarda, preserva la verdad, y en este sentido es fundamento (Cf. Gander 1994, pp. 24ss). Como vemos, el temple aparece en Heidegger como fundamento, por eso es temple fundamental, y así "la verdad no es nunca el 'sistema' articulado de propocisiones, a las que uno pudiera apelar, ella es el fundamento en cuanto reticente e invasor, que deja que sobresalga lo oculto sin suprimirlo, es el temple que ha sido afinado como tal fundamento (die als Grund stimmende Stimmung). Pues este fundamento es el Ereignis mismo como despliegue del Ser" (Heidegger 1989, p. 346). En este texto aparecen la verdad, el fundamento, el temple y Ereignis recubriéndose, no al modo de una identificación, pero sí referidos esencialmente uno al otro. 
La reserva aparecerá años más tarde como uno de los momentos del desasimiento, pero ya no se entenderá del mismo modo ni será conceptuada en términos de "temple fundamental". De algún modo, la "reserva misma" va transformándose en desasimiento (Gelassenheit) al que aludiremos ahora brevísimamente.

\section{El desasimiento ${ }^{1}$ (Die Gelassenheit)}

Sin querer aquí afirmar que el desasimiento es un temple fundamental, no podemos dejar de aludir, aunque sea brevemente, a aquel que para algunos (Gadamer 2003, p. 199) ocupa el sitial de la angustia en el Heidegger tardío.

En el "Debate en torno al lugar de la serenidad" de 1944-45 (no así en sus palabras pronunciadas en Messkirch en 1955), el desasimiento está caracterizado con un uso del lenguaje aparentemente no traducible o equivalente a la terminología propia del Heidegger tardío. Se hace mucho más difícil, por tanto, vislumbrar la figura del Ereignis en el desasimiento al modo como lo hemos intentado hacer respecto a la reserva. Abandonaremos anticipadamente la intención de establecer equivalencias entre la reserva y el Ereignis (y, en general, la terminología del segundo Heidegger) con el desasimiento y la región del encuentro, teniendo en claro también que se refieren de algún modo a lo mismo; incluso, como veremos, la reserva aparece como un momento del desasimiento. Lo que intentaremos hacer más bien es conceptuar el desasimiento como un intento de culminación de un único proyecto: la efectiva apertura a lo abierto, es decir, la experiencia del Ser en cuanto tal.

Ahora bien, el desasimiento no se entiende como un temple, en algunos pasajes es conceptuado como actitud -y en otros es negado como tal (Heidegger 1959, pp. 25 y 61)-, pero nunca es calificado como temple. Y es que, como quiero proponer aquí, el desasimiento pretende ser un momento unitario, del cual no cabe una distinción de facultades, es, si se quiere, una "voluntad-sapiente-atemperadora" (gestimmtwissenden-Willen), un querer (como un no-querer) de un corazón pensante (denkende Herz). De esta manera también pretende separarse radicalmente de lo que es una voluntad de dominio y de un pensar representativo. Lo veremos luego.

Antes de entrar propiamente en lo que es el desasimiento, hemos de referirnos al "lugar" de él, a la región del encuentro (die Gegnet) ${ }^{2}$, pues reluce en este lugar

1 Hay dos traducciones que recojo de Gelassenheit: Desasimiento (Carla Cordua) y Serenidad (Zimmermann). Ahora bien, la opción por alguna me parece un problema interpretativo pues en español la serenidad y el desasimiento mientan experiencias distintas. Se trata de comprender el análisis heideggeriano y localizar la experiencia a la que indica; allí se busca el término español. Mi opción ha sido por 'desasimiento', aunque a veces y en las citas tomadas de la traducción de Zimmermann aparece el término 'serenidad'.

2 Obviamente, esta expresión no pretende ser una traducción de Gegnet, sino simplemente una alusión más clara a dos de los elementos que hacen juego aquí: paraje o región y el venir a estar en contra. 
como un respecto, el que mira hacia nosotros, de esta región. La región del encuentro es aquella que, "lo reúne todo, en el demorar en el reposo en sí mismo, lo uno con lo otro y todo con cada uno, como si no pasara nada (sich nichts ereigne). Región del encuentro, es el cobijar desde atrás (Zurückbergen) que reúne para el amplio reposo en la morada" (Heidegger 1959, pp. 41s) La región del encuentro, como vemos, es la posibilitación de todo encuentro, no solo ni primeramente respecto a nosotros, sino como lo que reúne todo; no de un modo causal, sino como apertura del lugar de reunión, como amplitud y como espacio de tiempo que permite el reposar de toda cosa y su demora. Desde aquí, la región del encuentro deja brotar (aufgehen zu lassen) toda cosa en su reposado quedar, en su descanso (Ruhen). Es el respecto que da a las cosas. Desde este respecto "ella da la condición de cosa a la cosa (Sie bedingt das Ding zum Ding)" (Heidegger 1959, p. 56), es el cosificar (Bedingnis).

El otro respecto de la región del encuentro es el que da a nosotros, donde se sitúa el desasimiento. La relación entre la región del encuentro y nosotros (en el desasimiento) será el transcontrar (Vergegnis), entendiéndolo como "una relación de la contrada para con nosotros, que no sostenemos sino que dejamos que 'eso' nos ocurra" (Gadamer 2003, p. 190). Si el respecto de las cosas de esta región permite el reposar de toda cosa y su demora, su descanso; el respecto que da a nosotros también será el que nos deje brotar en nuestra esencia, nos deje descansar, por eso el desasimiento es una cierta quietud. "Consiste en que el hombre permanece dejado a la región del encuentro, bien es verdad que por obra de la región misma. El está en su esencia dejado a la región del encuentro en la medida en que pertenece originariamente a ésta. Le pertenece a ella en la medida en que está desde el inicio a-propiado ( $g e$ einet) a ella, y por cierto, por obra de la región del encuentro misma" (Heidegger 1959, p. 51).

Este desasimiento que no puede ser despertado por nosotros, no es ningún tipo de pasividad, el desasimiento se sitúa más allá de la diferenciación de actividad y pasividad y con ello queda fuera del dominio de la voluntad (Heidegger 1959, p.35). El desasimiento se constituye como un modo de no-querer, pero no se trata de que en el desasimiento no se quiera "algo", o ninguna cosa, sino más bien es un "soltarse" del querer mismo, es allí donde se descansa, donde se deja brotar la esencia del hombre como aquel que pertenece a la región del encuentro, el desasimiento es el dejarse apropiar por ella. El desasimiento es también un soltarse del pensar representativo, un desprenderse de todo representar, y con ello, la esencia del pensar queda (reposando) en el desasimiento. Pero, nos dice Heidegger, que el estar sueltos, liberados del querer y del representar, es solo el primer momento, que ni siquiera es necesario para el verdadero desasimiento, ya que la "verdadera serenidad puede acontecer sin que le preceda necesariamente esta liberación (Losgelassensein)" ". Hay que apuntar, pues,

3 En rigor, lo afirmado explícitamente aquí es la liberación respecto a la trascendencia horizóntica, pero claramente ella es una liberación del querer y el representar que la configura como tal. Afirmando más adelante, que el desasimiento es un soltarse del representar trascendental y prescindir del querer del horizonte. 
hacia aquel momento que toque de un modo más esencial al desasimiento. Es el desasimiento como espera (warten).

En efecto, el desasimiento es propiamente un reposar en la región del encuentro, que como tal nos introduce en la esencia del pensar, dejando brotar así la esencia misma del hombre. Aquello a partir de lo cual únicamente puede acontecer la experimentación de la esencia del pensar, es decir, la relación a la región del encuentro como lo abierto en cuanto tal, es en la espera. "El esperar es una relación para con la región del encuentro... tal vez sea incluso la relación, en la medida en que el esperar se introduce (einlassen) en la región del encuentro y, en este dejarse ir a su interior, hace que la región del encuentro impere puramente como región del encuentro" (Heidegger 1959 , p. 50). La espera es, dicho en otros términos, la apertura a lo abierto mismo, y lo abierto es la región del encuentro, "lo abierto mismo sería aquello respecto a lo cual puramente solo podemos esperar" (Heidegger 1959, p. 51), pues "en la espera dejamos abierto aquello que esperamos" (Heidegger 1959, p. 44). No se trata de tener una expectativa (erwarten) o una esperanza de algo, en donde nos representamos aquello que esperamos y nos atamos a ello. Mientras la experiencia desde el representar y la voluntad se hace a partir de objetos, "la espera no tiene propiamente objeto" (Heidegger 1959 , p. 44), por eso se templa ante lo abierto, es un permanecer confiado (überlassen) a la región del encuentro.

Pero hay otro momento más en el desasimiento, la insistencia (Inständigkeit) que consiste en su perseverar (Ausdauer), en el sostenerse de ella misma, como un recipiente que contiene y que es parte de lo que contiene. La perseverancia como un comportamiento (Verhalten) que se recoge en una "reserva (Verhaltenheit) que seguiría siendo en todo momento la reserva de la serenidad" (Heidegger 1959, p. 61). Esta reserva contiene y conserva el desasimiento, y el desasimiento puede así reposar en su esencia, que es la pertenencia a la región del encuentro.

Ahora bien, Heidegger nos dice a través de sus personajes y de "un poema copiado de alguna parte", que la insistencia es la nobleza del ánimo (Edelmut) y que esta nobleza constituye la esencia misma del pensar. Veamos: noble es aquello que "mora en el origen (Herkunft) de su esencia" (Heidegger 1959, p. 63); en este sentido, pensar es el saber de algún modo, rememorar (Andenken), que se pertenece a un origen, este origen es la región del encuentro. El pensar es esencialmente noble, porque pensando se sabe perteneciente a algo que de algún modo es anterior al pensar mismo y que en este sentido queda fuera de él (Unvordenklichen). La insistencia aquí es entonces precisamente el momento en que el pensar, como corazón pensante, "toca" su origen, por eso es noble, más bien dicho, por eso es un pensar de ánimo noble (Edelmut), paciente (Langmut) y generoso (Grossmut). Este es el ánimo del pensar sereno, del pensar esencial. 
Referencias bibliográficas

Gadamer, Hans-Georg (2003), Los caminos de Heidegger. Barcelona: Ed. Herder.

Gander, Hans-Helmut (1994), "Grund und Leitstimmungen in Heideggers 'Beiträge zur Philosophie", en Heidegger Studien. Volume 10. Berlin: Dunker \& Humbolt, pp. 15-32.

Heidegger, Martin (1959), Gelassenheit. Tübingen: Ed. Neske (Traducción de I. Zimmermann, 2002, Barcelona: Ed. del Serbal).

(1980), Hölderlins Hymnen. (Gesamtausgabe Band 39), Frankfurt a.M.: Vittorio Klostermann (Traducción inédita de Carolina Merino, Chile).

(1989), Beiträge zur Philosophie (Vom Ereignis). Frankfurt a.M: Vittorio Klostermann (Gesamtausgabe Band 65). (Traducción de B. Onetto, 2003, edición limitada, Valparaíso).

Rodríguez, Ramón (1997), La transformación hermenéutica de la filosofía. Madrid: Ed. Tecnos. 